УДК $512.54+512.55$

\title{
Local Automorphisms of Nil-triangular Subalgebras of Classical Lie Type Chevalley Algebras
}

Igor N. Zotov*

Institute of Mathematics and Computer Science

Siberian Federal University

Svobodny 79, Krasnoyarsk, 660041

Russia

Received 10.05.2019, received in revised form 10.07.2019, accepted 20.08.2019

We study the problem of describing local automorphisms of nil-triangular subalgebra of the Chevalley algebra over an associative commutative ring with identity.

Keywords: automorphism, local automorphism, standard central series, characteristic ideal, Chevalley algebra, nil-triangular subalgebra.

DOI: 10.17516/1997-1397-2019-12-5-598-605.

\section{Introduction}

A local automorphism of an algebra $A$ is arbitrary modular automorphism which acts on each element $\alpha \in A$ as suitable automorphism of this algebra. The local automorphisms of an algebra $A$ form a group under the composition of mappings (Lemma 1 in Section 1). Automorphisms of an algebra are its trivial local automorphisms.

Local automorphisms and local derivations of an algebra are systematically studied since the 1990s. According to [1], local automorphisms of the algebra $M(n, \mathbb{C})$ of complex $n \times n$ matrices exhausted by automorphisms and anti-automorphisms. See also [2] for local automorphisms of the simple Lie algebra $\mathfrak{s l}_{\mathfrak{n}}$ over a field of characteristic zero. R. Crist [3] constructed first example of a nontrivial local automorphism for subalgebra of triangular matrices in $M(3, \mathbb{C})$ with pairwise coincide elements on each diagonal.

Let $K$ be an associative commutative ring with identity. In [4] and [5] local automorphisms of the algebra $N T(n, K)$ of nil-triangular $n \times n$ matrices over $K$ and associated Lie algebra are investigated; they are described for $n=3$ and, when $K$ is a field, for $n=4$. In this article we study the more general problem of describing local automorphisms of nil-triangular subalgebra $N \Phi(K)$ of the Chevalley algebra over $K$ associated with a root system $\Phi$. The main result is a reduction Theorem 1 in Section 1. The proof of the theorem is devoted to Section 2. See also the remarks in Section 3.

\section{Remarks and the main theorem}

Further $K$ is an arbitrary associative commutative ring with identity, unless specified otherwise.

*zotovin@rambler.ru

(c) Siberian Federal University. All rights reserved 
According to [5] and [6], a local automorphism of an arbitrary $K$-algebra $A$ is an automorphism of the $K$-module $A$ which acts on each element $\alpha \in A$ as some automorphism depending, in general, on the choice of $\alpha$. (Definition in [1] has certain difference.) Local ring automorphisms are defined analogously. Denote by Laut $A$, the set of all local automorphisms of algebra $A$.

Lemma 1. The local automorphisms of any algebra A (similarly for the ring) form a group under the composition of mappings.

Proof. Choose an arbitrary $\phi, \psi \in$ Laut $A$. They acts on each element $x \in A$ as some automorphism $\phi_{x}, \psi_{x}$ of an algebra $A$. It is necessary to show that

$$
(\phi \psi)_{x}=\phi_{\psi(x)} \psi_{x}, \quad\left(\phi^{-1}\right)_{x}=\left(\phi_{\phi^{-1}(x)}\right)^{-1}, \quad x \in A
$$

It's evident that, $x=\phi(z)=\phi_{z}(z)$ with uniquely $z \in A$. Hence

$$
\begin{gathered}
\phi^{-1}(x)=z=\phi_{z}^{-1}\left(\phi_{z}(z)\right)=\left(\phi_{z}\right)^{-1}(x)=\left(\phi^{-1}\right)_{x}(x), \\
(\phi \psi)(x)=\phi\left(\psi_{x}(x)\right)=\phi_{\psi_{x}(x)}\left(\psi_{x}(x)\right)=\left(\phi_{\psi(x)} \psi_{x}\right)(x)=(\phi \psi)_{x}(x) .
\end{gathered}
$$

This completes the proof for local ring automorphisms. From here lemma follows easily.

We investigate local automorphisms of a nil-triangular subalgebra in Chevalley $K$-algebras.

A Chevalley algebra over a field $K$ is associated with each indecomposable root system $\Phi$ in the Euclidean space and characterized by Chevalley base consisting of generating elements $e_{r}$ $(r \in \Phi)$ [7, Sec. 4.4]. We fix a base $\Pi$ in $\Phi$. Positive system of roots $\Phi^{+} \supseteq \Pi$ in $\Phi$ is unique [7]. The subalgebra $N \Phi(K)$ with the base $\left\{e_{r} \mid r \in \Phi^{+}\right\}$is said to be a niltriangular subalgebra.

According to Chevalley's theorem on base [7, Sec. 4.2], if $r, s \in \Phi^{+}$, then

$$
e_{r} * e_{s}=N_{r, s} e_{r+s}=-e_{s} * e_{r}(r+s \in \Phi), \quad e_{r} * e_{s}=0(r+s \notin \Phi),
$$

where either $N_{r, s}= \pm 1$ or $|r|=|s|<|r+s|$ and $N_{r, s}= \pm 2$ or $\Phi$ is of type $G_{2}$ and $N_{r, s}= \pm 2$ or \pm 3 . The signs of the structure constants $N_{r, s}$ may be chosen arbitrarily (up to isomorphisms $N \Phi(K))$ for extraspecial pairs $(r, s) \in \Phi^{+}$, [7, Proposition 4.2.2].

The height of the root $r$ is the sum $h t(r)$ of the coefficients in the expansion of $r$ in the base $\Pi$ in $\Phi$. The Coxeter number $h=h(\Phi)$ of the system $\Phi$ equals $h t(\rho)+1$, where $\rho$ is a maximal root in $\Phi^{+},[7,8]$. Subalgebras $L_{m}$ with base $\left\{e_{r} \mid r \in \Phi^{+}, h t(r) \geqslant m\right\}$ form in the algebra $L_{1}=N \Phi(K)$ the standard central series

$$
L_{1} \supset L_{2} \supset \cdots \supset L_{h-1}=K e_{\rho} \supset L_{h}=0, h=h t(\rho)+1 .
$$

We now may to formulate our main theorem.

Theorem 1. The ideal $L_{2}$ of Lie algebra $N \Phi(K)$ of classical type of rank $>4$ is characteristic and any local automorphism of $N \Phi(K)$ acts as its suitable automorphism, modulo $L_{2}$.

\section{Proof of the main theorem}

It is well known that the standard central series (1) of the algebra $N \Phi(K)$ is an upper central (or hypercentral) and lower central, except the cases $2 K \neq K$, when the system $\Phi$ has no roots of different lengths and, also, the case $6 K \neq K$ for type $G_{2}$. Thus, all ideals $L_{m}$ in the Lie algebra 
$N \Phi(K)$ are characteristic when all roots of the system $\Phi$ have the same length or $2 K=K$ for the types $B_{n}, C_{n}$ and $F_{4}$.

The Lie algebra $N \Phi(K)$ of type $A_{n-1}$ is associated to the algebra $N T(n, K)$ of all lower nil-triangular (with zeros on and above the main diagonal) $n \times n$ matrices over $K$. Usual matrix units $e_{i j}(1 \leqslant j<i \leqslant n)$ gives Chevalley base $\left\{e_{r} \mid r \in \Phi^{+}, e_{r}=e_{i j}\right\}$ after the corresponding numbering of the roots.

The Lie algebras $N \Phi(K)$ of type $B_{n}, C_{n}$ and $D_{n}$ are given in [9] similarly in the base of matrix units $e_{i v}$, respectively

$$
-i<v<i \leqslant n, \quad-i \leqslant v<i \leqslant n, v \neq 0, \quad 1 \leqslant|v|<i \leqslant n .
$$

We assume that $r=r_{i v}$ as $e_{r}=e_{i v}$. The sums of two roots that are the root, in addition to the standard $r_{i j}+r_{j v}=r_{i v}$, as for the type $A_{n}$, here also $r_{k v}+r_{m,-v}=r_{k,-m}(k>m>|v|)$ and for type $C_{n}$, moreover, $r_{k v}+r_{k,-v}=r_{k,-k}(k>|v|)$. Any element of the Lie algebra $N \Phi(K)$ here is represented by a $\Phi^{+}$-matrix ||$a_{i v} \mid=\sum a_{i v} e_{i v}$ for corresponding type. Thus, the $B_{n}^{+}$-matrix has the form

$$
\begin{aligned}
& a_{10} \\
& \begin{array}{lll}
a_{2,-1} & a_{20} & a_{21}
\end{array} \\
& \text {... } \ldots \ldots \\
& \begin{array}{lllllll}
a_{n,-n+1} & \ldots & a_{n,-1} & a_{n 0} & a_{n 1} & \ldots & a_{n, n-1} .
\end{array}
\end{aligned}
$$

If we cancel zeros column, then we obtain $D_{n}^{+}$-matrix.

Let $T_{i m}$ be the ideal of all $\Phi^{+}$-matrices of $\left\|a_{u v}\right\|$ with the condition $a_{u v}=0$ for $u<i$ or $v>m$. We assume that $T_{1 m}:=T_{i m}$ if for the selected $\Phi$ and $m$ the number $i$ is the smallest. In the Lie algebra $N \Phi(K)$ of type $B_{n}\left(\right.$ or $\left.N B_{n}(K)\right)$, we select submodules $R_{j}:=\sum_{i=j}^{n} K e_{i 0}$, $1 \leqslant j \leqslant n$, and also select the submodule $L_{j}^{[0}$ with base $\left\{e_{u v} \mid 0 \leqslant v<u \leqslant n, u-v \geqslant j\right\}$.

We need the following two lemmas from [10].

Lemma 2. Let $2 K \neq K$ and $n \geqslant 2$. Then the Lie rings $N B_{n}(K)$ and $N C_{n}(K)$ generate

$$
\begin{gathered}
\left\{K e_{i i-1}(1 \leqslant i \leqslant n) ; \quad K e_{2,-1}\right\}, \\
\left\{K e_{i i-1}(2 \leqslant i \leqslant n) ; \quad K e_{i,-i}(1 \leqslant i \leqslant n)\right\},
\end{gathered}
$$

respectively, and no $K e_{i v}$ can be dropped in them.

Denote by $\mathcal{A}_{2}$, the annihilator of the element 2 .

Lemma 3. Hypercenters of the Lie algebra $N C_{n}(K)(n \geqslant 2)$ are written as

$$
Z_{i}=L_{2 n-i}+\mathcal{A}_{2} L_{2 n-i-1}(1 \leqslant i<2 n-1), \quad Z_{2 n-1}=L_{1} .
$$

For the algebra $N B_{n}(K)(n \geqslant 2)$ we have

$$
\begin{aligned}
& Z_{i}=L_{2 n-i}+\mathcal{A}_{2} R_{n+1-i}(1 \leqslant i \leqslant n-2), \quad Z_{n-1}=L_{n+1}+\mathcal{A}_{2} R_{2}+\mathcal{A}_{2} e_{n 1} \\
& Z_{n+i}=L_{n-i}+\mathcal{A}_{2} R_{1}+\mathcal{A}_{2} L_{n-i-2}^{[0} \quad(0 \leqslant i \leqslant n-3), \quad Z_{2 n-2}=L_{2}+\mathcal{A}_{2} L_{1} .
\end{aligned}
$$


The diagonal automorphisms $h(\chi): e_{r} \rightarrow \chi(r) e_{r}\left(r \in \Phi^{+}\right)$of the Lie algebra $N \Phi(K)$ correspond to each $K$-character $\chi$ of the root lattices to the multiplicative group $K^{\sharp}$ of invertible elements of the ring $K$ [7, Sec. 7.1].

For any root $r$ the mapping $t \rightarrow x_{r}(t):=\exp \left(t \cdot a d . e_{r}\right)(t \in K)$ generate an isomorphism of the additive group $K^{+}:=(K,+)$ to the automorphism group of the algebra Chevalley. Root subgroups $X_{r}=x_{r}(K)$ generate a Chevalley group, [7,11]. The restrictions of automorphisms of its unipotent subgroup $U \Phi(K)=\left\langle X_{r}\left(r \in \Phi^{+}\right)\right\rangle$generate the subgroup $J$ of inner automorphisms of the Lie algebra $N \Phi(K)$.

The standard automorphisms of the Lie algebra $N \Phi(K)$ include inner, diagonal, graph [7, Ch. 12] and central automorphisms, that is, the identity automorphism modulo the center.

According to [9], if the Lie ring (or group) does not coincide with its $m$ th hypercenter, then its automorphism is said to be hypercentral of height $m$, or simply hypercentral, if it is the identity automorphism modulo the $m$ th hypercenter and an outer automorphism modulo the $(m-1)$ th hypercenter.

The main hypercentral automorphisms of height $>1$ of Lie algebras of $N \Phi(K)$ of classical types are revealed previously, [12-14]. Let $V(\Phi, K)$ denote the subgroup generated by them.

It is well known that the adjoint group of the ring $R=N T(n, K)$ under the adjoint multiplication $a \circ b=a+b+a b$ is isomorphic to the unitriangular group $U T(n, K)$. The automorphism group of the associated Lie algebra $\Lambda(R)$ (that is, $N \Phi(K)$ of type $A_{n-1}$ ) is found in [12]:

$$
\text { Aut } \Lambda(R)=\mathcal{Z} \cdot J \cdot V \cdot \mathcal{D} \cdot W \quad(n>4),
$$

where $\mathcal{Z}, \mathcal{D}$ and $W$ are subgroups of central, diagonal and idempotent automorphisms, respectively. The subgroup $V$ is generated by the main hypercentral automorphisms of height 2 and for $\mathcal{A}_{2} \neq 0$ - of height 3 . For the Lie ring $\Lambda(R)$ the subgroup $\simeq$ Aut $K$ of induced automorphisms is added, [12, Theorem 1]. The description of automorphisms in [12] also for $n=3,4$ is certain difference.

In the Lie algebra $N \Phi(K)$ of type $B_{n}$, the ideal $L_{2}$ is larger than the commutant as $2 K \neq K$, by Lemma 2 and Lemma 3 . When $\mathcal{A}_{2} \neq 0$, it less than hypercenter $Z_{2 n-2}$. The Lie algebra $N B_{n}(K)$ admits hypercentral automorphisms, whose height depends linearly on the rank $n$. To any pair $t, d \in \mathcal{A}_{2}$ there corresponds such automorphism

$$
\chi_{t, d}: \alpha \rightarrow \alpha+\sum_{k=2}^{n-1} a_{k,-1}\left(t e_{k 0}+d e_{n,-k}\right),
$$

which translates the (-1)th column of the $B_{n}^{+}$-matrix to 0th column.

The subgroup in Aut $N B_{n}(K)$ isomorphic to the adjoint group in $\mathcal{A}_{2}$ form semi-diagonal automorphisms

$$
\delta_{c}^{(-1)}: e_{k v} \rightarrow(1+c) e_{k v}(0<-v<k \leqslant n), e_{k v} \rightarrow e_{k v}(0 \leqslant v<k \leqslant n),
$$

with invertible $1+c \in 1+\mathcal{A}_{2}$.

According to [13], for simple symmetric roots $r$ and $\bar{r} \neq r(\overline{\bar{r}}=r)$ of a system roots $\Phi$ of type $D_{n}(n \geqslant 4)$, an isomorphic embedding ${ }^{\sim}$ of the subgroup

$$
S=\left\{\alpha=\left\|a_{u v}\right\| \in S L(2, K): 2 a_{11} a_{12}=2 a_{21} a_{22}=0\right\}
$$

of the group $S L(2, K)$ into the group Aut $N D_{n}(K)$ is defined by the rule

$$
\widetilde{\alpha}: \quad e_{r} \rightarrow a_{11} e_{r}+a_{12} e_{\bar{r}}, \quad e_{\bar{r}} \rightarrow a_{21} e_{r}+a_{22} e_{\bar{r}}, \quad e_{s} \rightarrow e_{s} \quad(s \in \Pi \backslash\{r, \bar{r}\}) .
$$


The description of automorphisms of Lie rings $N \Phi(K)$ of classical types is completed in [13]. It is summarized by the following theorem.

Theorem 2. Any automorphism of the Lie ring $N C_{n}(K)(n>4)$ is a product of the standard and hypercentral of $V(\Phi, K)$ automorphisms. For the Lie ring $N B_{n}(K)(n>4)$, a semi-diagonal automorphism is added as a factor, and for the Lie ring $N D_{n}(K)(n>4)$ an automorphism of $\widetilde{S}$ is added as a factor.

Remark that the ideal $L_{3}$ is not even invariant under the hypercentral automorphism $\chi_{t, d}$. On the other hand, we have the following lemma.

Lemma 4. The ideal $L_{2}$ in the Lie algebra $N \Phi(K)$ of rank $>4$ of the classical type is always characteristic.

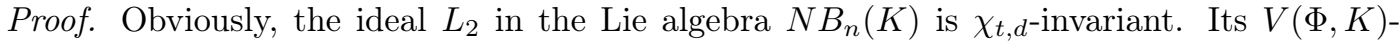
invariance follows directly from the definitions of the other main hypercentral automorphisms of height $>1$ in all cases with regard to the restriction on Lie rank, $[13,14]$.

Graph automorphisms of Lie algebras $N \Phi(K)$ of rank $>4$ are defined only for root systems of the same length (more precisely, for $A_{n}, D_{n}$ and $E_{n}$ types, $n=6,7,8$ ). As noted above, in these cases all ideals of $L_{m}$ are characteristic. With respect to diagonal (and ring) automorphisms, all one-dimensional subalgebras $K e_{r}$ are invariant, and therefore any $L_{m}$ ideals are invariant. This is also true for semi-diagonal automorphisms of the Lie algebra $N B_{n}(K)$.

The inner automorphisms act on $L_{m}$ identically modulo $L_{m+1}$, which also implies $J$-invariance of all $L_{m}$. Taking into account that under the conditions of the lemma the center $Z_{1}$ always is in $L_{2}$, we obtain the characteristic of the ideal $L_{2}$ with respect to any standard automorphism. This completes the proof of the lemma.

The first statement of main theorem 1 is given by Lemma 4 . Let us prove the second statement.

The Lie algebra $N \Phi(K)$ of type $A_{n-1}$ is represented, as above, by the algebra $\Lambda(R)$ for $R=N T(n, K)(n>4)$. The group automorphisms Aut $\Lambda(R)$ is factorized by the product $(2)$, and its normal subgroup $\mathcal{Z} \cdot J \cdot V$ acts identically modulo $L_{2}$.

Let $i^{\prime}:=n+1-i$. According to [12], any idempotent $g$ of the ring $K$ defines a Lie automorphism

$$
\tau_{g}: e_{i j} \rightarrow g e_{i j}+(-1)^{i-j-1}(1-g) e_{j^{\prime} i^{\prime}}, 1 \leqslant j<i \leqslant n,
$$

called idempotent and $W=<\tau_{g} \mid g \in K, g^{2}=g>$. In particular, for the case $i^{\prime}=i+1$ we obtain the characteristic ideal

$$
T_{i^{\prime} i^{\prime}-1}=\tau_{0}\left(T_{i+1 i}\right)=T_{i+1 i}
$$

of the Lie algebra $\Lambda(R)$.

Any local automorphism $\varphi$ of the Lie algebra $\Lambda(R)$ acts on an arbitrary element modulo $L_{2}$ as a suitable automorphism of $\mathcal{D} \cdot W$. Taking into account that $\varphi$ is $K$-module automorphism, we have, up to its multiplication by an automorphism of $\mathcal{D} \cdot W$,

$$
\varphi\left(e_{21}\right)=e_{21}, \quad \varphi\left(x e_{21}\right)=x \varphi\left(e_{21}\right)=x e_{21} \quad \bmod L_{2} \quad(x \in K) .
$$

We need the following lemma (cf. [15, Lemma 1.3.5]).

Lemma 5. If $\varphi\left(e_{21}\right)=e_{21}$ for a local automorphism $\varphi$ of Lie algebra $\Lambda(R)(n>4)$, then

$$
\varphi\left(e_{i+1 i}\right) \in T_{i+1 i}+L_{2}, \quad 1 \leqslant i<n .
$$


Proof. Assume that the inclusion (3) is violated for some number $i, 1<i<n$ and $i^{\prime} \neq i+1$. Taking into account the condition $n>4$, for some idempotent $f \neq 1$ we obtain, up to a multiplication of $\varphi$ by a diagonal automorphism, the following equalities:

$$
\varphi\left(e_{21}\right)=e_{21}, \quad \varphi\left(e_{i+1 i}\right)=\tau_{f}\left(e_{i+1 i}\right) \quad \bmod R^{2}, \quad 1-f \neq 0 .
$$

Since there exists automorphism $\psi \in A u t \Lambda(R)$ acting on $e_{21}+e_{i+1 i}$ similarly to $\varphi$, so

$$
e_{21}+f e_{i+1 i}+(1-f) e_{i^{\prime} i^{\prime}-1}=\psi\left(e_{21}\right)+\psi\left(e_{i+1 i}\right) \bmod L_{2} .
$$

We can assume that $\psi \in D \cdot W$ and hence $\psi=\delta \tau_{g}$ for a suitable idempotent $g$ and diagonal automorphism $\delta$. If $i<n-1$, then $(n, n-1)$-projection of the element (4) on the right is in $(1-g) K^{\sharp}$, and on the left is zero. Hence $g=1$ and $\psi=\delta$. Comparing now $\left(i^{\prime}, i^{\prime}-1\right)$-projections of matrices in (4) on the right and left, we obtain the equality $1-f=0$, that gives a contradiction.

It remains to investigate the case $i=n-1$. Using (4), we find invertible elements $c, d \in K$ such that

$$
\begin{gathered}
(2-f) e_{21}+f e_{n n-1}=\left(g e_{21}+(1-g) e_{n n-1}\right)^{\delta}+\left(g e_{n n-1}+(1-g) e_{21}\right)^{\delta}, \\
(2-f) e_{21}+f e_{n n-1}=(c g+c(1-g)) e_{21}+(d(1-g)+d g) e_{n n-1} .
\end{gathered}
$$

The last equality gives $d=f$. It is easily follows $f=1$. This contradicts to the condition $f \neq 1$.

As corollary of the proved lemma we obtain the equalities for some elements $c_{i} \in K$

$$
\varphi\left(e_{i+1 i}\right)=c_{i} e_{i+1 i} \quad\left(\bmod L_{2}\right)
$$

Now the equality (2) shows that $\varphi$ acts on each element of $e_{i+1 i}$ modulo $L_{2}$ as a suitable automorphism of $\mathcal{D}$. It follows that all elements of $c_{i}$ are invertible in $K$. This completes the proof of the theorem for the type $A_{n}$.

Any local automorphism of the $\varphi$ Lie algebra $N D_{n}(K)$ acts on arbitrary element, as a suitable automorphism. Up to multiplication by an automorphism, one can even assume that the equality $\varphi\left(e_{2,-1}\right)=e_{2,-1}$ is satisfied.

Then $\varphi$ acts modulo $L_{2}$ on each element $e_{i+1 i}$ as a suitable automorphism from the product $\mathcal{D} \cdot \widetilde{S}$. Therefore

$$
\begin{gathered}
\varphi\left(e_{21}\right)=a_{21} e_{2,-1}+c_{1} e_{21} \quad \bmod L_{2}, \\
\varphi\left(e_{i+1 i}\right)=c_{i} e_{i+1 i} \quad \bmod L_{2}, \quad 2 \leqslant i<n,
\end{gathered}
$$

where all elements of $c_{i}(1 \leqslant i<n)$ are invertible in $K$ and $2 a_{21}=0$. Up to multiplication of $\varphi$ by a diagonal automorphism, we can assume that $c_{i}=1(1 \leqslant i<n)$. Then $\varphi$ acts modulo $L_{2}$ as an automorphism $\widetilde{\alpha}$ with matrix

$$
\alpha=\left(\begin{array}{cc}
1 & 0 \\
a_{21} & 1
\end{array}\right) .
$$

Further we note that if an ideal of an algebra is characteristic, then it is invariant with respect to any local automorphism of the algebra. From the Theorem 2 easily implies the following lemma

Lemma 6. In the Lie ring $N C_{n}(K)(n>4)$, the ideals $T_{i j}$ for $i<n$ and the ideals $T_{i v}$ for $v<0$ are characteristic. 
It follows from lemma that the ideal $T_{2,-2}$ is characteristic in the Lie algebra $N C_{n}(K)(n>4)$ and this shows that any local automorphism of $\varphi$ induces a local automorphism of the factor algebra

$$
N C_{n}(K) / T_{2,-2} \simeq N A_{n}(K) \simeq N T(n+1, K)
$$

moreover, $\varphi\left(T_{1,-1}\right)=T_{1,-1}$. Applying the proved case for the type $A_{n}$, we obtain the statement of the theorem for the type $C_{n}$.

In the Lie ring $N B_{n}(K)(n \geqslant 5)$ the ideals $T_{10}$ and $T_{10}+T_{21}$ are always characteristic and

$$
N B_{n}(K) / T_{10} \simeq N A_{n-1}(K) \simeq N T(n, K) .
$$

Applying the proved case for the type $A_{n}$, we obtain the statement of theorem for the type $B_{n}$.

The theorem is proved.

\section{Some remarks}

The algebra $R$ is called enveloping for the Lie algebra $L$ if replacing the multiplication in $R$ with new $a * b:=a b-b a$ gives algebra $R^{(-)}$isomorphic to $L$. It's obvious that Aut $R \subseteq A$ t $R^{(-)}$, [16]. Unlike the Lie algebras $N \Phi(K)$ the enveloping algebras (in general, non-associative) that are constructed for them in $[16,17]$ depend on the choice of signs of the constants $N_{r, s}$.

When the choice of signs of the constants of the Lie algebra $N \Phi(K)$ of the classical type corresponds to its representation in [9], the enveloping algebra is denoted by $R \Phi(K)$. The developed methods are applicable for transferring the main theorem to algebras $R \Phi(K)$.

The restrictions in the main theorem on the rank of $n$ are related to the fact that some basic hypercentral automorphisms of the Lie algebra $N \Phi(K)$ of classical Lie type for small $n$ can remain automorphisms that are not hypercentral automorphisms. In these cases, the action of Aut $N \Phi(K)$, modulo $L_{2}$, becomes exceptional. See [4,12] for the type $A_{n}$ and the description of Aut $N D_{4}(K)$ in $[9]$.

The author thanks professor V. M. Levchuk for statement of a problem and attention to the work.

\section{References}

[1] D.R.Larson, A.R.Sourour, Local derivations and local automorphisms of B(H), Proc. Sympos. Pure Math., 51(1990), 187-194.

[2] T.Becker, J.Escobar Salsedo, C.Salas, R.Turdibaev, On local automorphisms of $\mathfrak{s l}_{\mathfrak{n}}$, arXiv:1711.11297, 2018.

[3] R.Crist, Local automorphisms, Proc. Amer. Math. Soc., 128(2000), 1409-1414.

[4] A.P.Elisova, Local automorphisms of nilpotent algebras of matrices of small orders, Russian Mathematics (Iz. VUZ), 57(2013), no. 2, 40-48 (in Russian).

[5] A.P.Elisova, I.N.Zotov, V.M.Levchuk, G.S.Suleimanova, Local automorphisms and local derivations of nilpotent matrix algebras, The Bulletin of Irkutsk State University. Series Mathematics, 4(2011), no. 1, 9-19 (in Russian). 
[6] I.N.Zotov, Local Automorphisms of Nilpotent Algebras of Matrices of Small Orders, Conference thesis of XLII regional student scientific conference of mathematics and computer science, Krasnoyarsk, SibFU, 2009, 24-25 (in Russian).

[7] R.W.Carter, Simple groups of Lie type, New York, Wiley and Sons, 1972.

[8] N.Bourbaki, Groupes et algebraes de Lie (Chapt. IV-VI), Hermann, Paris, 1968.

[9] V.M.Levchuk, Automorphisms of unipotent subgroups of Chevalley groups, Algebra And Logic, 29(1990), no. 3, 315-338 (in Russian).

[10] I.N.Zotov, V.M.Levchuk, The Mal'tsev correspondence and isomorphisms of niltriangular subrings of Chevalley algebras, Trudy Inst. Mat. i Mekh. UrO RAN, 24(2011), no. 4, 135-145 (in Russian).

[11] C.Chevalley, On Some Simple Groups, Matematika, Periodic Collection of Translations of Foreign Papers, 2(1958), no. 1, 3-53 (in Russian).

[12] V.M.Levchuk, Connections between a unitriangular group and certain rings. Part 2. Groups of automorphisms, Siberian Mat. J., 24(1983), 543-557 (in Russian).

[13] V.M. Levchuk, A.V. Litavrin, Hypercentral automorphisms of nil-triangular subalgebras in Chevalley algebras, Sib. Elektron. Mat. Izv., 13(2016), 467-477 (in Russian).

[14] A.V.Litavrin, Automorphisms of nil-triangular subrings of classical Chevalley algebras. Diss. cand. phys.-math. sciences. Tomsk, TSU, 2017 (in Russian).

[15] A.P.Elisova, Local automorphisms and local derivations of nilpotent algebras, Diss. cand. phys.-math. sciences. Krasnoyarsk, SibFU, 2013 (in Russian).

[16] V.M.Levchuk, The Niltriangular Subalgebra of the Chevalley Algebra: the Enveloping Algebra, Ideals, and Automorphisms, Doklady Mathematics, 97(2018), no. 1, 23-27.

[17] V.M.Levchuk, Niltriangular subalgebra of Chevalley algebra and the enveloping algebras, Group Theory in Ankara, Middle East Technical University, 2019, 13-14.

\section{Локальные автоморфизмы нильтреугольных подалгебр алгебр Шевалле классических типов}

Игорь Н. Зотов

Институт математики и фундаментальной информатики Сибирский федеральный университет Свободный, 79, Красноярск, 660041

Россия

Исследуется задача описания локальных автоморбизмов нильтреугольной подалгебры алгебры Шевалле над ассочиативно-коммутативным кольцом с единицей.

Ключевые слова: автоморфизм, локальный автоморфизм, стандартный центральный ряд, характеристический идеал, алгебра Шевалле, нилътреугольная подалгебра. 